\title{
Modeling dispersion of partial discharges due to propagation velocity variation in power cables
}

\author{
J. Granado ${ }^{\mathrm{a}, *}$, A. Torralba ${ }^{\mathrm{a}}$, C.Álvarez-Arroyo ${ }^{\mathrm{b}}$ \\ Universidad de Sevilla. Descubrimientos s/n. 41092-Sevilla (Spain) \\ ${ }^{a}$ Department of Electronic Engineering \\ ${ }^{b}$ Department of Electrical Engineering
}

\begin{abstract}
Existing models for partial discharge (PD) propagation based on a single attenuation constant are unable to explain how each frequency component travels with a different propagation velocity. This paper proposes a new model based on a complex propagation term whose real component does not depend on the frequency $(f)$, and whose imaginary part is modeled with a second order polynomial in $f$. The proposed model explains how the $\mathrm{PD}$ is attenuated, delayed, and dispersed due to the fact that each frequency component is differently delayed.

A closed-form expression is proposed for the PD peak value and width, and a method to derive the model parameters from a reference model existing in the bibliography. Simulation results show that the peak value and width of the propagated PD pulse are similar to those obtained with the proposed model. Additionally, the proposed model provides the velocity of each PD frequency component, which is crucial to get an accurate estimation of the PD source location.

The parameters of the proposed model have been estimated using a vector network analyzer for a XLPE cable. These results have been compared to the measurement obtained in a medium voltage test bench where intentionally induced PDs have been captured and processed, confirming the results of attenuation, delay and dispersion predicted by the proposed model.
\end{abstract}

Keywords: Partial discharges, propagation model, distribution MV cables.

\section{Introduction}

The observation of the phenomenon known as partial discharge (PD) in cables allows engineers to predict imminent failures in medium voltage (MV) distribution networks. Locating PD sources allows to identify the cable degraded areas and, preventively, replace it to avoid a power outage that could affect hundreds of subscribers.

To get an accurate location of the area where partial discharges are being generated, it is necessary to know how these PDs propagate along the cable. Published propagation models are based on classical transmission line (TL) approaches [1-5]. But these models have the following disadvantages:

- It is difficult to estimate their parameters, mainly due to the cable complex structure, as well as the lack of knowledge about the high frequency behavior of its materials [2].

- Existing propagation models for partial discharges, including those based on transmission lines, usually yield to a nearly constant propagation velocity $[2,6]$. However, experimental results shown in [1] or [2] demonstrate that this velocity depends on the PD frequency components.

\footnotetext{
${ }^{*}$ Corresponding author: Tel./Fax $+34954481303 /+34954487373$

Email addresses: j_granado@us.es (J. Granado), torralba@us.es (A. Torralba), cesaralvar@gmail.com (C.Álvarez-Arroyo)
}

This dependence leads to dispersion, i.e the pulse spreads because each frequency component travels at a different propagation velocity [7]. It is well-known that dispersion has a significant effect on the pulse shape [8], as well as negative implications for PD location using techniques such as Time Domain Reflectometry (TDR) [3]. Dispersion has also negative effect on PD pattern classification and recognition [9].

- Understanding how the propagation velocity varies with frequency, or even with cable aging, is crucial for an accurate location of the PD source [5].

Dispersion is mainly due to the high frequency behavior of the semiconducting layer [8]. In addition, small variations of the characteristic impedance along the cable, especially due to aging, also leads to dispersion [5].

Dispersion is modeled in the bibliography by means of a TL model where the propagation term is a real value that linearly depends on the frequency [10]. Thus, dispersion appears because the high frequency components are highly attenuated. Consequently, the signal bandwidth is reduced, and the original PD shape is spread in the time domain. Since the propagation term is real, this model is unable to explain how each spectral component travels at a different speed.

Present techniques for fault location use those less attenuated spectral components to estimate the fault location. As the fre- 
quency components could change, even from experiment to experiment, due to changes in external conditions, or due to cable aging, assuming the same propagation velocity for every frequency component can yield a non-negligible error in the fault location.

This paper proposes a new propagation model for medium voltage cables. This model can be used to study the propagation of PDs, or even other transient phenomenon (lighting impulses, switching transients or breakdowns). It is based on the contributions of Marcuse in the field of light pulse propagation in singlemode optical fibers [11]. The proposed model approaches the attenuation term as frequency invariant, since the useful parts of the spectrum of the signals measured at the cable ends exhibit a low dependence with frequency. The phase term is approximated by a second order polynomial in frequency. This approximation allows to explain how the propagation velocity varies with frequency leading to dispersion.

A simple method valid for both, symmetric and asymmetric pulses, is proposed in this paper to estimate the model parameters from the reference model in [10]. The results obtained with the proposed model are compared by simulation to those obtained with the model of that reference.

The main advantage of the proposed model is that the model parameters can be estimated from experimental measurements, and two methods are proposed here. The first one uses a vector network analyzer (VNA) to measure the frequency response of a cable sample. In the second technique, partial discharges are captured in a degraded cable and digitally processed to measure the pulse dispersion due to cable propagation. Experimental results obtained with a XLPE MV cable are shown to illustrate these methods.

This paper is organized in 5 additional sections. Section 2 describes the proposed propagation model for both, symmetric and asymmetric pulses. Section 3 describes how to extract the model parameters from the reference model described in [10]. Section 4 illustrates how the proposed model agrees with the experimental measurements. In section 5 we discuss some key aspects related to location of partial discharges that can be addressed with the proposed model. Finally, some conclusions are drawn in section 6 .

\section{Proposed propagation model}

A transfer function $H(\omega, L)$, shown in (1), is proposed to model the propagation of PDs in MV cables. In (1), $\gamma(\omega)$ is the complex propagation term, and $L$ is the propagated distance.

$$
\begin{array}{r}
H(\omega, L)=\exp (-\gamma(\omega) L) \\
\text { where: } \gamma(\omega)=\alpha_{0}+j \beta(\omega) .
\end{array}
$$

The real part of the propagation term, $\alpha_{0}$, is responsible for PD power reduction in $8.68 \alpha_{0} \mathrm{~dB} / \mathrm{m}$. A typical value for $\alpha_{0}$ is $0.03 \mathrm{~dB} / \mathrm{m}$.

Concerning the imaginary part of the propagation term, or phase term $\beta(\omega)$, it models the phase variation due to propagation. Term $\beta(\omega)$ determines the propagation velocity, the propagation delay, and is responsible for pulse dispersion. Unlike most of the models proposed in the literature, which assume $\beta(\omega)$ to have a linear dependence with $\omega$, the model proposed here assumes a quadratic dependence with $\omega$ as shown in (2).

$$
\begin{gathered}
\beta(\omega)=\beta_{0}+\beta_{1} \omega+(1 / 2) \beta_{2} \omega^{2}, \\
\text { where: } \beta_{1}=\partial \beta /\left.\partial \omega\right|_{w=0} \text { and } \beta_{2}=\partial^{2} \beta /\left.\partial \omega^{2}\right|_{w=0} .
\end{gathered}
$$

Delay constant $\beta_{1}$ is responsible for the delay (also called group delay) of the initial pulse after travelling a distance $L$. The propagation time $t_{p}=\beta_{1} L$ can be considered to be the mean value of the propagation delay of every spectral components in the propagated pulse. Constant $\beta_{2}$, or dispersion constant, is responsible for the spreading of the initial pulse shape due to the fact that each spectral component propagates at a different speed.

\subsection{Gaussian pulses}

Expression (3) models an initial gaussian pulse with amplitude and deviation $A_{0}$ and $\sigma_{0}$, respectively. Figure 4(a) shows this signal. Using the proposed propagation model, the resulting propagated pulse at a distance $L$ is shown in (4), where it can be observed that: a) the resulting pulse is gaussian too; b) it has been be attenuated $\left(A_{L}\right.$ is the resulting peak value and even if we consider a non dissipative $\left(\alpha_{0}=0\right)$ medium, the peak value would be reduced due to dispersion if $\beta_{2} \neq 0$ ); c) it has been phase delayed in an amount of $\theta_{L}$ radians; d) it has been time delayed $t_{p}$ seconds; and e) it has been dispersed along the cable: the original deviation $\sigma_{0}$ has grown up to $\sigma_{L}$ due to dispersion.

$$
\begin{aligned}
& v(t, 0)=A_{0} \exp \left(-t^{2} / 2 \sigma_{0}^{2}\right) . \\
& v(t, L)=A_{L} \exp \left(-j \theta_{L}\right) \exp \left(-\left(t-t_{p}\right)^{2} / 2 \sigma_{L}^{2}\right),
\end{aligned}
$$

where:

$$
\begin{aligned}
& \sigma_{L}=\left(\sigma_{0}^{2}+\beta_{2}^{2} L^{2} / \sigma_{0}^{2}\right)^{1 / 2} \\
& A_{L}=A_{0} \sigma_{0}\left(\sigma_{0}^{4}+\beta_{2}^{2} L^{2}\right)^{-1 / 4} \exp \left(-\alpha_{0} L\right), \\
& \theta_{L}=\beta_{0} L+\arctan \left(\beta_{2} L / 2 \sigma_{0}^{2}\right)-\ldots \\
& \beta_{2} L / 2\left(\sigma_{0}^{4}+\beta_{2}^{2} L^{2}\right), \text { and } \\
& t_{p}=\beta_{1} L
\end{aligned}
$$

\subsection{Asymmetric pulses}

The skewed nature of the PD pulse is modeled in [10] by means of a sum of delayed gaussian functions. Using $N_{G}$ components, the resulting pulse waveform is given in (5), where $A_{0, k}, \sigma_{0, k}$, and $\tau_{0, k}$ are the peak, typical deviation, and delay values of the gaussian component $k$, respectively. Figure 6(a) shows the asymmetric pulse generated using this model.

$$
v(t, 0)=A_{p} \sum_{k=1}^{N_{G}} A_{0, k} \exp \left(-\left(t-\tau_{0, k}\right) /\left(2 \sigma_{0, k}^{2}\right)\right),
$$

where:

$$
\begin{aligned}
& A_{0, k}=0.2245 \exp (-k / 10), \\
& \tau_{0, k}=10^{-9}(k+5000), \text { and } \\
& \sigma_{0, k}=10^{-9} \exp \left(k^{0.25}\right) / \sqrt{2} .
\end{aligned}
$$




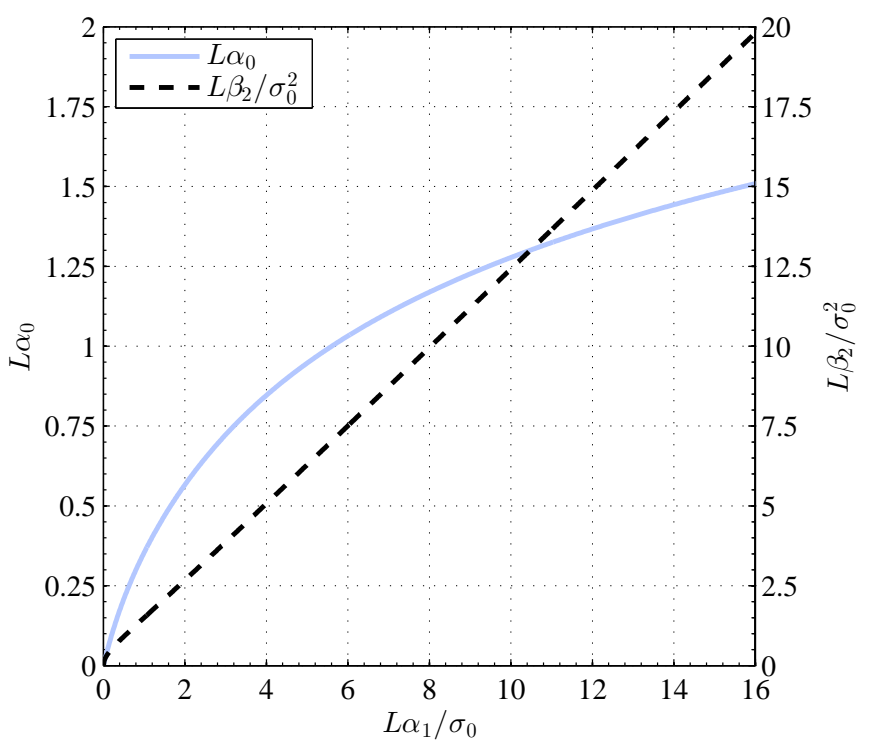

Figure 1: Relationship between the proposed and the Oussalah model: $L \alpha_{0}$ and $L \beta_{2} / \sigma_{0}^{2}$ are the adimensional attenuation and dispersion constants of the proposed model, respectively. $L \alpha_{1} / \sigma_{0}$ is the attenuation constant of the model defined in [10].

Assuming that the propagation medium is linear, the expression in (6) describes the $L$ meter propagated asymmetric pulse, where $\alpha_{0}, \beta_{1}$, and $\beta_{2}$ are the attenuation, delay, and dispersion constants of the proposed method. The resulting $v(t, L)$ signal is composed of $N_{G}$ gaussian components, where $A_{L, k}, \sigma_{L, k}$, and $\tau_{L, k}$ are the amplitude, deviation, and delay of the $k$-th component, respectively.

$$
v(t, L)=A_{p} \sum_{k=1}^{N_{G}} A_{L, k} \exp \left(-\left(t-\tau_{L, k}\right) /\left(2 \sigma_{L, k}^{2}\right)\right),
$$

where:

$$
\begin{aligned}
& A_{L, k}=A_{0, k} \exp \left(-\alpha_{0} L\right) \sigma_{0, k}\left(\sigma_{0, k}^{4}+\beta_{2}^{2} L^{2}\right)^{-1 / 4}, \\
& \sigma_{L, k}^{2}=\sigma_{0, k}^{2}+\left(\beta_{2} L / \sigma_{0, k}\right)^{2} \\
& \tau_{L, k}=\tau_{0, k}+\beta_{1} L .
\end{aligned}
$$

\section{Model fitting}

This section proposes a procedure to estimate the attenuation $\left(\alpha_{0}\right)$ and dispersion $\left(\beta_{2}\right)$ constants of the proposed model, from the (also called) attenuation constant $\left(\alpha_{1}\right)$ of the model proposed by Oussalah et al. in [10]. Let's remind that the Oussalah model considers a real propagation term $\gamma(\omega)=\alpha_{1} \omega$, where parameter $\alpha_{1}$ is called the attenuation constant.

The dispersion constant $\beta_{2}$ of our model is computed by equalling the expressions for the deviation $\sigma_{L}$ in both models. The numerical solution in a graphical way is shown in Figure 1 , where the adimensional dispersion constant $\left(L \beta_{2} / \sigma_{0}^{2}\right)$ of the proposed model is depicted versus the adimensional attenuation constant $\left(L \alpha_{1} / \sigma_{0}\right)$ of the Oussalah model.

The expressions of the peak values $\left(A_{L}\right)$ are used in both models to derive the attenuation constant $\alpha_{0}$. Figure 1 also

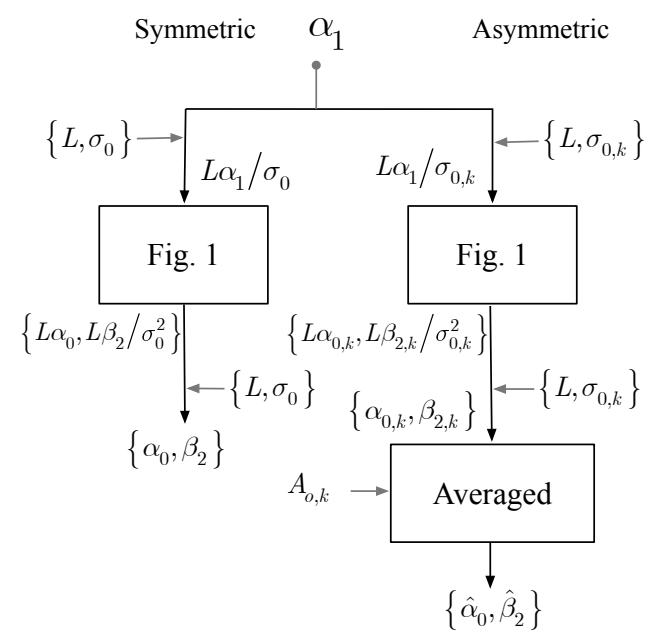

Figure 2: Flowchart to estimate the attenuation $\left(\alpha_{0}\right)$ and dispersion $\left(\beta_{2}\right)$ constants of the proposed model from the (also called) attenuation constant $\alpha_{1}$ proposed in [10]. Two different flows are shown for the symmetric and asymmetric pulses.

shows the adimensional attenuation constant $\left(L \alpha_{0}\right)$ of the proposed model versus the adimensional attenuation constant $\left(L \alpha_{1} / \sigma_{0}\right)$ of the reference one. Note that, in order to estimate $\alpha_{0}$, we need the previously estimated value of $\beta_{2}$. The delay constant $\beta_{1}$ cannot be derived from the Oussalah model since the propagation delay is ignored in that approach.

The following subsections describes the procedure to obtain the attenuation and dispersion constants for both, symmetric and asymmetric pulses. The flow diagram of Figure 2 summarizes the proposed procedure.

\subsection{Symmetric pulse}

Once $\alpha_{1}$ is known, and accounting for the initial deviation $\sigma_{0}$ that determines the spectral components of the original pulse, the following procedure is proposed to estimate the model parameters for the symmetric case (see Figure 2):

1. Compute the adimensional attenuation constant of the Ousalah model, choosing any value of $L$ in the abscissa of Figure 1. For example, for a MV cable with $\alpha_{1}=$ $4 \mathrm{e}-11 \mathrm{~s} \mathrm{~m}^{-1}$, using a gaussian pulse with $\sigma_{0}=2 \mathrm{~ns}$, at $L=300 \mathrm{~m}$, the resulting adimensional attenuation constant of the Oussalah model is $L \alpha_{1} / \sigma_{0}=6$. The selected value for $\sigma_{0}$ is especially important to estimate $\alpha_{0}$, and it is related to the transient phenomenon under study. The selected one is widely used for partial discharges in cables.

2. The parameters of the proposed model will be estimated using Figure 1. For the above example, the resulting value of the dispersion and attenuation adimensional constants are, respectively: $L \beta_{2} / \sigma_{0}^{2}=7.50$, and $L \alpha_{0}=1.03$. Then, the estimated model parameters are: $\alpha_{0}=3.44 \mathrm{e}-3 \mathrm{~m}^{-1}$ and $\beta_{2}=0.10 \mathrm{~ns}^{2} \mathrm{~m}^{-1}$.

\subsection{Asymmetric pulses}

As the constants $\alpha_{0}$ and $\beta_{2}$ are conditioned by the initial pulse deviation $\sigma_{0}$, we will derive as many values of these 


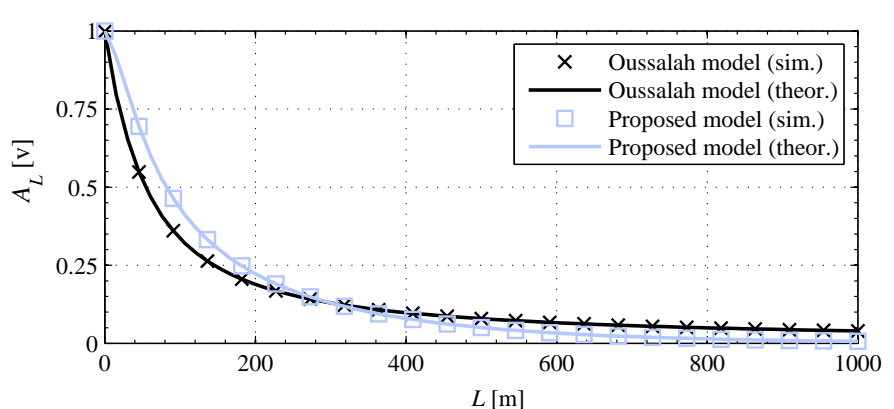

(a)

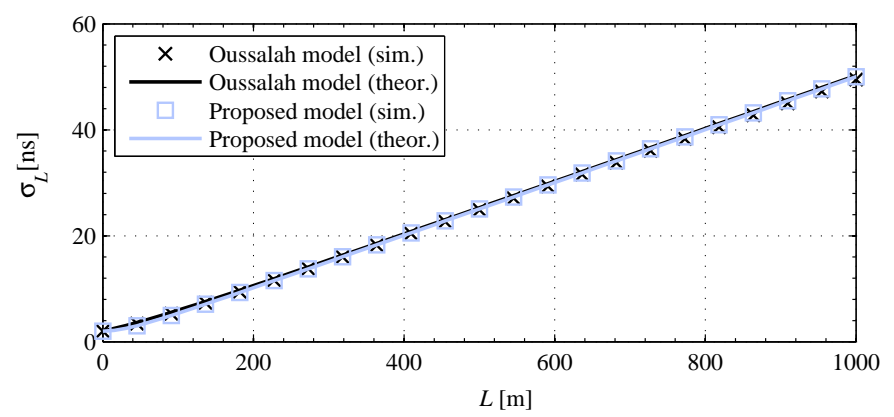

(b)

Figure 3: Simulation of:(a) peak value, and (b) deviation, versus distance for symmetric pulse propagation.

constants as the number of gaussian components used to initially build the asymmetrical pulse. Let $\alpha_{0, k}$ and $\beta_{2, k}$ be the propagation constants for the $k$-th component of the proposed model derived with the same constant $\alpha_{1}$, and using the initial deviations $\sigma_{0, k}$. For example: using a MV cable with $\alpha_{1}=$ $4 \mathrm{e}-11 \mathrm{sm}^{-1}$, the resulting attenuation constants are in the interval $\alpha_{0,1}=3.5 \mathrm{e}-3 \mathrm{~m}^{-1}$ to $\alpha_{0,60}=1.22 \mathrm{e}-3 \mathrm{~m}^{-1}$, while the dispersion constants are in the range from $\beta_{2,1}=9.62 \mathrm{e}-2 \mathrm{~ns}^{2} \mathrm{~m}^{-1}$ to $\beta_{2,60}=6.78 \mathrm{e}-1 \mathrm{~ns}^{2} \mathrm{~m}^{-1}$.

A simplified model for the asymmetric pulse propagation is also proposed here where the $N_{G}$ pairs of propagation constants $\left\{\alpha_{0, k}, \beta_{2, k}\right\}$ are replaced by a single pair of averaged propagation constants $\left\{\hat{\alpha}_{0}, \hat{\beta}_{2}\right\}$. They are computed as shown in (7) and (8), where the propagation constants have been averaged and weighted with the component amplitude of each gaussian component $A_{0, k}$. The resulting values for the example above are: $\hat{\alpha}_{0} \simeq 2.54 \mathrm{e}-3 \mathrm{~m}^{-1}$ and $\hat{\beta}_{2} \simeq 0.21 \mathrm{~ns}^{2} \mathrm{~m}^{-1}$

$$
\begin{aligned}
& \hat{\alpha_{0}}=\sum_{k=1}^{N_{G}} \alpha_{0, k} A_{0, k} / \sum_{k=1}^{N_{G}} A_{0, k} \\
& \hat{\beta_{2}}=\sum_{k=1}^{N_{G}} \beta_{2, k} A_{0, k} / \sum_{k=1}^{N_{G}} A_{0, k}
\end{aligned}
$$

\subsection{Simulation results}

The propagation of a gaussian pulse has been simulated in a cable using both, the transfer function proposed in this paper,

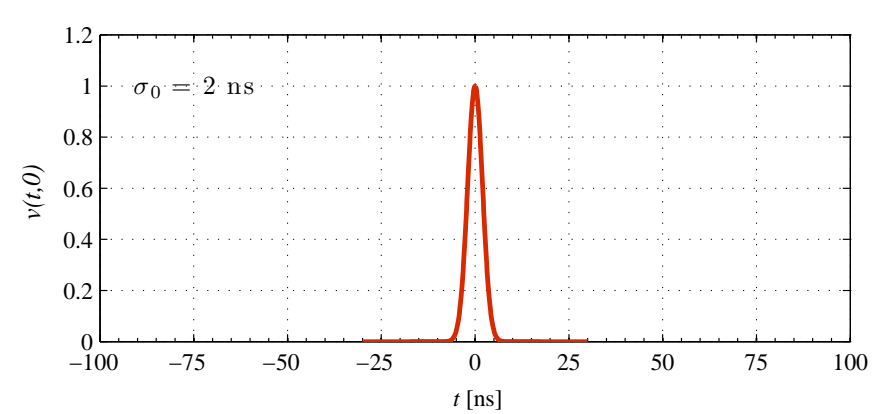

(a)

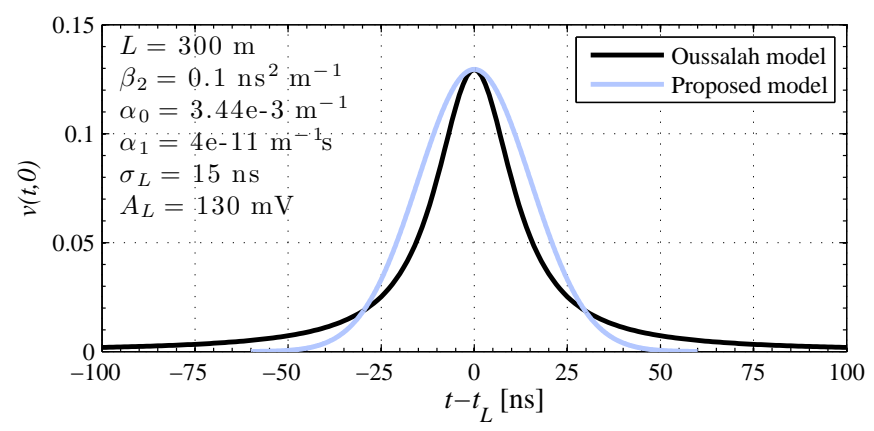

(b)

Figure 4: Example of symmetric pulse propagation: (a) initial pulse, (b) pulse shape after 300 meters of propagation. $t_{L}=t_{p}(L=300)$ is the propagation delay.

and the model presented in [10]. The initial peak value of the gaussian pulse is $A_{0}=1 \mathrm{~V}$, and its initial deviation is $\sigma_{0}=2 \mathrm{~ns}$. The proposed model is configured with $\alpha_{0}=3.4 \mathrm{e}-3 \mathrm{~m}^{-1}$, and $\beta_{2}=0.1 \mathrm{~ns}^{2} \mathrm{~m}^{-1}$. The Oussalah model uses $\alpha_{1}=4 \mathrm{e}-11 \mathrm{~m}^{-1} \mathrm{~s}$. Figure 3 draws the peak value $A_{L}$ and deviation $\sigma_{L}$ versus $L$, for the proposed and the Oussalah models. For comparison purposes this figure also shows the theoretical behavior of those variables given in (4) and [10].

To obtain the simulated results, the value of the transfer function in every model was computed for every distance $L$; this value was multiplied by the Fourier transform of the initial pulse, and the resulting function was anti-transformed to the time domain. Finally, the peak and deviation values were estimated by means of the gaussian function that fits the best the simulation results. Note that the Oussalah model provides a non-gaussian shaped function.

Simulated peak values depicted in Figure 3(a) shows that: 1) simulation and theoretical results are very similar, even though the Oussalah model provides a pulse which is symmetrical but not gaussian; and 2) both simulated models perform similarly, especially for $L>400 \mathrm{~m}$. Furthermore, the values of the deviation $\sigma_{L}$ for both models, obtained by simulation, are shown in Figure 3(b), along with their theoretical values. This figure shows that these four values (theoretical and simulated ones for each model) are almost equal in the distance interval analyzed here.

For the sake of visual comparison, Figure 4(b) shows the shape of the initial pulse (in Figure 4(a)) after having travelled 


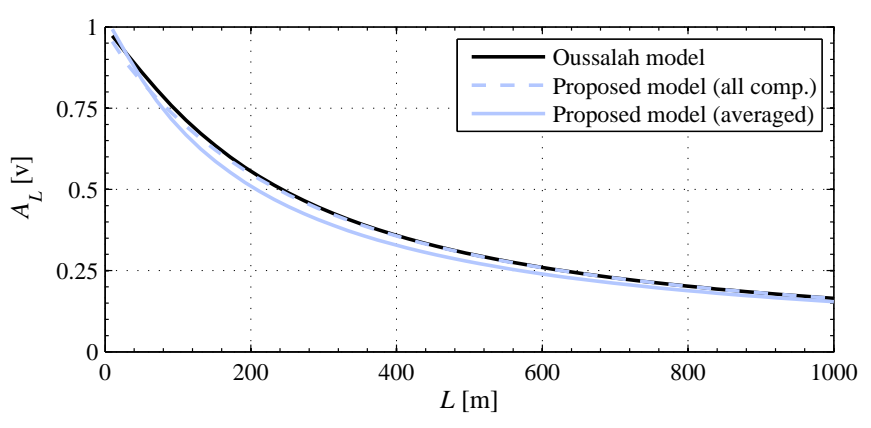

(a)

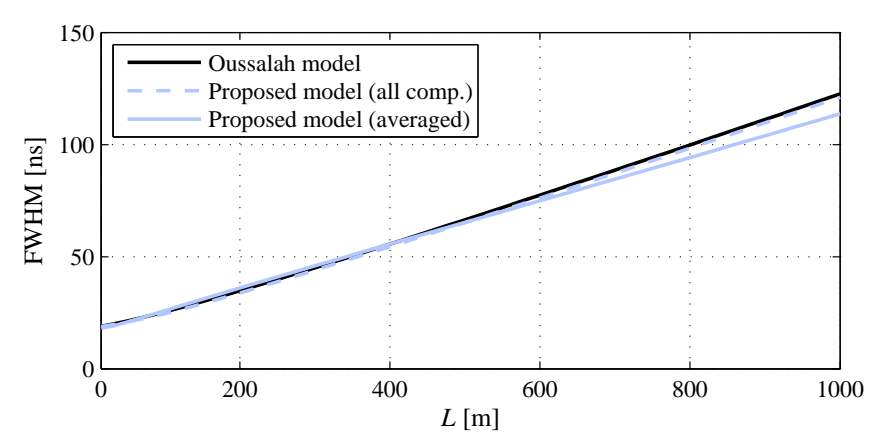

(b)

Figure 5: Simulation of: (a) peak value, and (b) FWHM, versus distance for asymmetric pulse propagation.

300 meters, for both, the proposed and the Oussalah models. In this figure we can see that both pulses have the same peak value $(0.13 \mathrm{~V})$, and the same deviation $\left(\sigma_{L}=15 \mathrm{~ns}\right)$. The pulse shape of the proposed model is gaussian while the other one is symmetric but not gaussian.

In order to validate the proposed model for asymmetric pulses, the propagated pulse is rebuilt from the resulting amplitudes and deviations of the $k$-th gaussian component, $A_{L, k}$ and $\sigma_{L, k}$, respectively. To obtain the propagation constants, the method proposed in Section 3 has been used with, both, a set of $N_{G}$ propagation constants $\left\{\alpha_{0, k}, \beta_{2, k}\right\}$, and a single pair of averaged propagation constants $\left\{\hat{\alpha}_{0}, \hat{\beta}_{2}\right\}$. Once the pulse has been rebuilt, its peak and width values were estimated. To compute the pulse width, the FWHM (Full Width Half Maximum) value has been used, due to the asymmetric nature of the pulse shape. For $L$ high enough, the pulse becomes symmetric, so that it can be approximated by a gaussian function. In that case, $\mathrm{FWHM} \approx 2.35 \sigma$, where $\sigma$ is the gaussian pulse deviation.

The peak value $A_{L}$ and the FWHM pulse width versus distance $L$ are shown in Figure 5, for both, the model described in [10] and the proposed model. We can observe that both models behave almost identical, even when the averaged propagation constants $\hat{\alpha}_{0}$ and $\hat{\beta}_{2}$ are used.

Figure 6 shows the shapes of an initially asymmetric pulse that propagates according to the proposed model and the model of reference [10]. In Figure 6 (a) we can see the initial asymmetric pulse, with a FWHM pulse width of $18 \mathrm{~ns}$ and a peak value of $1 \mathrm{~V}$. Figure 6(b) depicts the symmetric pulse shape

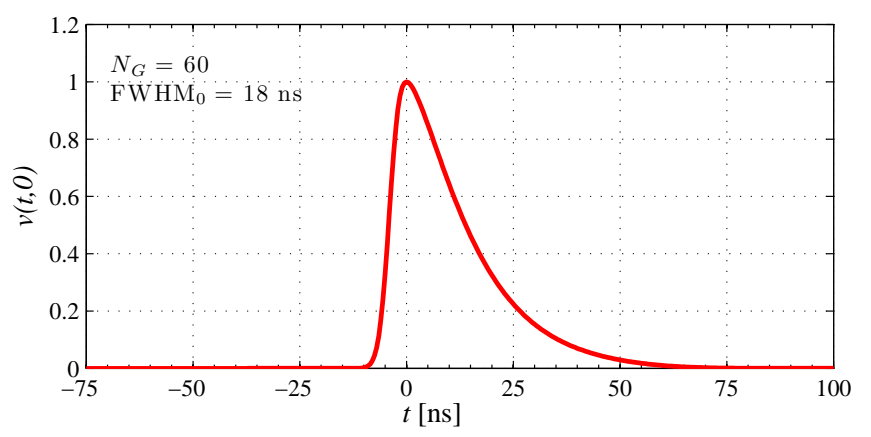

(a)

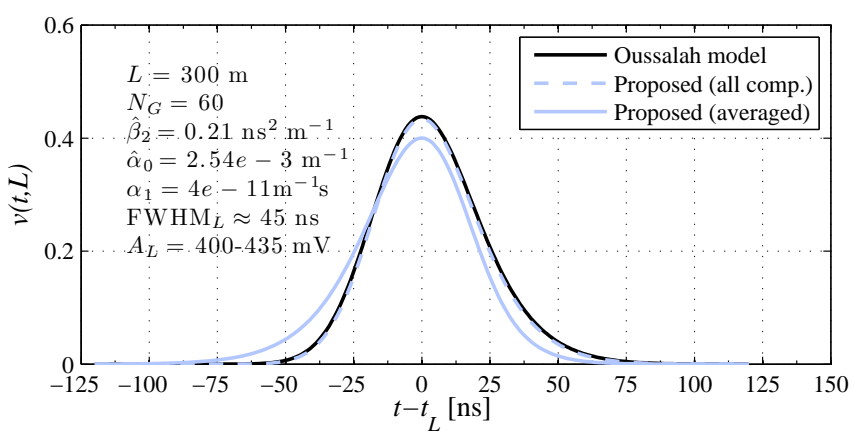

(b)

Figure 6: Example of asymmetric pulse propagation: (a) initial pulse, (b) pulse shape after 300 meters of propagation. $t_{L}=t_{p}(L=300)$ is the propagation delay.

after having been propagated $L=300 \mathrm{~m}$, using the proposed model with the full set of propagation constants, the proposed model with the averaged value of the propagation constants, and the Oussalah model. The proposed model provides a pulse shape similar to the results obtained in [10]. Slight differences can found in the peak value $(0.40 \mathrm{vs}$. 0.43$)$ when the averaged model is used, but the resulting FWHM pulse widths have a similar value (44 ns) in any case.

\section{Experimental results}

\subsection{VNA measurements}

Using a VNA the scattering parameter $S_{21}$ of a $42.5 \mathrm{~m}$ long new MV XLPE cable has been measured, in order to estimate the attenuation term $\alpha(f)$ and the phase term $\beta(f)$ of its transfer function. The lower bound of the frequency interval is set to $1 \mathrm{MHz}$, as the current transformers used to measure the partial discharges reject lower frequency components. Despite PD spectral components can initially reach up to $\mathrm{GHz}$, propagation makes the highest components to become extinct in a few meters [8]. Setting the upper limit to $50 \mathrm{MHz}$, a proper frequency range is selected.

The experimental results have been fitted in the minimum mean squared error sense, regarding the estimation of the attenuation, delay, and dispersion constants $\left(\alpha_{0}, \beta_{1}, \beta_{2}\right)$ for the proposed model, and the attenuation constant $\left(\alpha_{1}\right)$ for the model of reference [10]. These results are shown in Table 1 . Note that 
the measured dispersion constant $\beta_{2}$ is negative, indicating that the group propagation velocity increases with frequency.

A similar MV cable was simulated in [8], providing a velocity versus frequency curve. Selecting some points of this curve, we have estimated $\beta_{1}$ in the interval $[5.7,6.2] \mathrm{ns} / \mathrm{m}$, which decreases with frequency in the spectral interval under study, and $\beta_{2}=-0.796 \mathrm{~ns}^{2} \mathrm{~m}^{-1}$. Simulation results obtained in that paper are close to those experimentally obtained here; moreover, the value estimated for $\alpha_{1}$ is also similar to that reported in [10] for XLPE cables.

By following the procedure described above, the measured $\alpha_{1}$ is now used to adjust the proposed model. Using an initial deviation $\sigma_{0}=5 \mathrm{~ns}$, we obtain $\alpha_{0}=4.40 \mathrm{e}-3 \mathrm{~m}^{-1}$ and $\left|\beta_{2}\right|=0.491 \mathrm{~ns}^{2} \mathrm{~m}^{-1}$. Note that the proposed method is unable to resolve whether the sign of the dispersion constant is positive or negative.

\subsection{Partial discharge measurements}

In this section we describe the results obtained in a medium voltage test bench, where a XLPE cable was stressed at operating conditions $(18 \mathrm{kV})$ without load. The dielectric was artificially damaged in a small region close to one of the cable ends until partial discharges appeared. These PDs were acquired at both cable ends, and properly processed to estimate the model parameters.

\subsubsection{Scheme of reference}

The diagram of Figure 7 shows the scheme of reference. Two current transformers were installed at both cable ends. These components are broadband inductive sensors that are responsible for sensing the PD pulses. According to standard [12], the selected sensor was an ultra wide band coupler.

The cable was degraded by drilling holes that affected the thermoplastic sheath, the semiconducting layer, and the insulation. In Figure 7, the degraded area was located $L_{1}$ meters from one of the ends, labeled as Near End, and $L_{2}$ meters from the other one (labeled as Far End). As shown in Figure 7, $L_{2}>>L_{1}$ is assumed.

In Figure $7 x(t, 0)$ is the original PD signal, and signals $x\left(t,-L_{1}\right)$ and $x\left(t, L_{2}\right)$ are the signals captured by sensors located at the near and far ends, respectively. Both signals are acquired synchronously using a digital oscilloscope triggered by the near end signal. The sample frequency was set to $500 \mathrm{MHz}$, and a proper dynamic range for each channel was selected. Captured signals were stored to be off-line processed.

Table 1: Summary of experimental results: using a vector network analyzer, and measuring the PDs as described in section 4.2

\begin{tabular}{lcccc} 
Method & $\begin{array}{c}\alpha_{1} \\
{\left[\mathrm{~s} \mathrm{~m}^{-1}\right]}\end{array}$ & $\begin{array}{c}\alpha_{0} \\
{\left[\mathrm{~m}^{-1}\right]}\end{array}$ & $\begin{array}{c}\beta_{1} \\
{\left[\mathrm{~ns} \mathrm{~m}^{-1}\right]}\end{array}$ & $\begin{array}{c}\beta_{2} \\
{\left[\mathrm{~ns}^{2} \mathrm{~m}^{-1}\right]}\end{array}$ \\
\hline VNA & $5.16 \mathrm{e}-11$ & $3.37 \mathrm{e}-3$ & 5.72 & -0.7121 \\
$\begin{array}{l}\text { Direct PD } \\
\text { measure }\end{array}$ & $\mathrm{n} / \mathrm{a}$ & $3.40 \mathrm{e}-3$ & 5.92 & \pm 0.761 \\
\hline
\end{tabular}

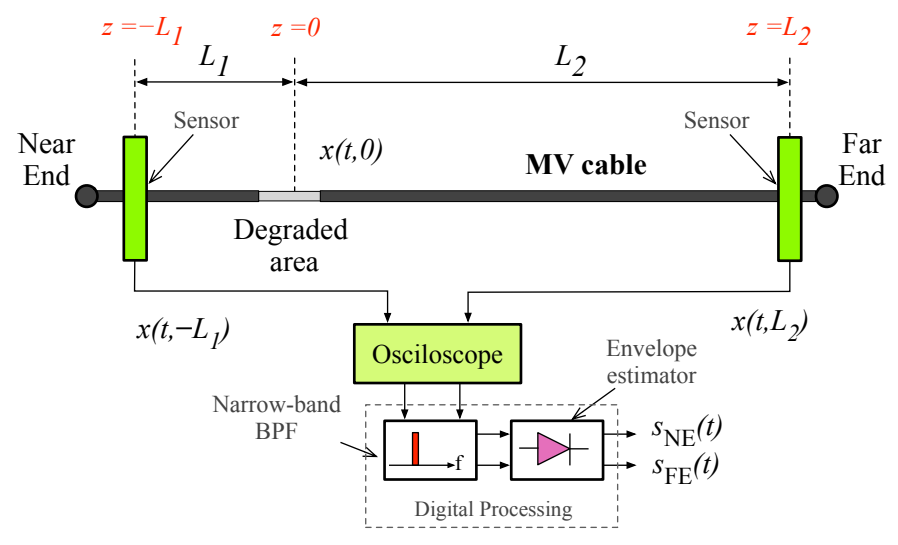

Figure 7: Test bench description, and definition of main variables

\subsubsection{Digital processing}

Digital processing was used to estimate the delay and dispersion due to propagation along the cable of a small spectral interval of the PD signal. A narrow band pass filter (NB-BPF) is used whose center frequency $f_{c}$ is tunable. The selected impulse response is finite and gaussian, providing a linear phase response. When the wide-band signal $x\left(t,-L_{1}\right)$ is filtered, the resulting spectrum is also gaussian, centered in the same frequency, and with the same bandwidth as the filter. On the other hand, when the far end signal $x\left(t, L_{2}\right)$ is filtered, we should obtain a signal whose frequency domain spectrum is conformed by the cable response as well as by the filter. The resulting signals are shifted to the baseband from $f_{c}$ by means of an envelope estimation. Thus, signals $s_{N E}(t)$ and $s_{F E}(t)$ are the baseband near end and far end envelopes, respectively.

\subsubsection{Experimental estimation of $\alpha_{0}, \beta_{1}$, and $\beta_{2}$}

The attenuation, delay, and dispersion constants of the proposed model can be extracted from the baseband signals $s_{N E}(t)$ and $s_{F E}(t)$. The solution is only valid for a small frequency interval around the center frequency $f_{c}$. The linear effects coming form sensors, capture system, and digital processing are unbiased since they affect in the same way to both signals.

To compute the attenuation constant expression (9) was used, where $P_{N E}$ and $P_{F E}$ are the powers of signals $s_{N E}(t)$ and $s_{F E}(t)$, respectively. To estimate the delay constant, the delay between the near and far end envelopes $\left(\Delta t_{d}\right)$ was detected using a simple cross-correlation method. Constant $\beta_{1}$ was calculated according to (10).

$$
\begin{aligned}
& \alpha_{0}=\ln \left(P_{N E} / P_{F E}\right) / 2\left(L_{2}-L_{1}\right) \\
& \beta_{1}=\Delta t_{d} /\left(L_{2}-L_{1}\right)
\end{aligned}
$$

We can estimate the dispersion constant $\beta_{2}$ in two different ways:

1. By measuring the delay constant $\beta_{1}$ for each center frequency $f_{c}$. Then, $\beta_{2}$ can be estimated by computing the derivative of $\beta_{1}$ with respect to frequency. This technique is inaccurate, as $\beta_{2}$ has not a significant effect on 
the propagation delay. For example, for a $300 \mathrm{~m}$ long cable with $\beta_{2}=0.5 \mathrm{~ns}^{2} \mathrm{~m}^{-1}$, and considering a spectral interval of $5 \mathrm{MHz}$, the group delay is expected to shift $\pm(0.5 \cdot 2 \pi \cdot 5 \mathrm{e} 6) \cdot 300= \pm 4.7 \mathrm{~ns}$. That is, less than 3 samples using the sampling frequency of our test bench.

2. We can also estimate the dispersion constant by measuring the PD dispersion, i.e. measuring the spreading of the PD pulse due to propagation along the cable. This dispersion is also observed in signals $s_{N E}(t)$ and $s_{F E}(t)$. This technique was chosen here to estimate $\beta_{2}$.

Expression (11) shows the proposed formulae for the deviation of the near and far end baseband signals $\left(\sigma_{N E}\right.$ and $\sigma_{F E}$, respectively). In this expression, $\sigma_{0}$ is the initial PD deviation (assuming it is gaussian), $\sigma_{s}$ is the dispersion due to the sensor used to capture the PD signal (we assume that its impulse response is also gaussian and similar in both ends), and $\sigma_{N B P F}$ is the deviation of the gaussian impulse response of the narrow band pass filter (equal in both ends). It should be noted that the digital filters do not disperse the PD as their phase response is linear but, as its frequency response is band-limited, they will spread the incoming pulses.

Although in (11) $\sigma_{0}$ is unknown, it can be indirectly estimated using an additional signal measurement: the signal captured at the cable near end. The deviation of this signal $\left(\sigma_{1}\right)$ is approximated in (13). Combining (12) and (13) we obtain an expression for the initial deviation $\sigma_{0}$ in (14). This approximation is valid when $L_{1}$ is large enough so as to make the captured pulse to become symmetrical, and assuming that the impulse response of the selected sensor also behaves gaussian.

$$
\begin{aligned}
\sigma_{N E}^{2} & =\sigma_{0}^{2}+\left(\beta_{2} L_{1} / \sigma_{0}\right)^{2}+\sigma_{s}^{2}+\sigma_{N B P F}^{2}, \\
\sigma_{F E}^{2} & =\sigma_{0}^{2}+\left(\beta_{2} L_{2} / \sigma_{0}\right)^{2}+\sigma_{s}^{2}+\sigma_{N B P F}^{2} . \\
\sigma_{F E}^{2}-\sigma_{N E}^{2} & =\left(L_{2}^{2}-L_{1}^{2}\right)\left(\beta_{2} / \sigma_{0}\right)^{2} \\
\sigma_{1}^{2} & =\sigma_{0}^{2}+\left(\beta_{2} L_{1} / \sigma_{0}\right)^{2}+\sigma_{s}^{2} \\
\sigma_{0}^{2}=\sigma_{1}^{2} & -\sigma_{s}^{2}-\left(\sigma_{F E}^{2}-\sigma_{N E}^{2}\right)\left(L_{1}^{2} /\left(L_{2}^{2}-L_{1}^{2}\right)\right)
\end{aligned}
$$

\subsubsection{Results}

The test bench of Figure 7 has been built with $L_{1}=1.5 \mathrm{~m}$ and $L_{2}=304 \mathrm{~m}$. The cable was artificially damaged until a high frequency activity due to PDs in the degraded area was observed. The center frequency was tuned to $f_{c}=3 \mathrm{MHz}$ with a FWHM bandwidth of $1 \mathrm{MHz}$ (the equivalent time response deviation is $\sigma_{B P F}=0.37 \mu \mathrm{s}$ ). This center frequency was selected because the signal level measured at the far end was high enough when compared to other spectral components.

The FWHM bandwidth of the selected current transformer, measured with a VNA, was $50 \mathrm{MHz}$ (the equivalent time domain deviation is $\sigma_{s}=7.51 \mathrm{~ns}$ ). It was observed that frequency components bellow $1 \mathrm{MHz}$ were highly attenuated.

During the test campaign, thousands of partial discharges were captured. Each test sequence provided about 4610 captures that were considered as valid, and they were later processed to estimate the proposed model parameters.

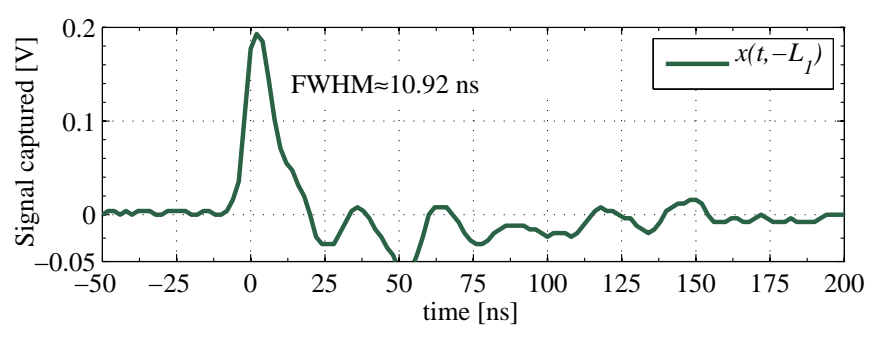

(a)

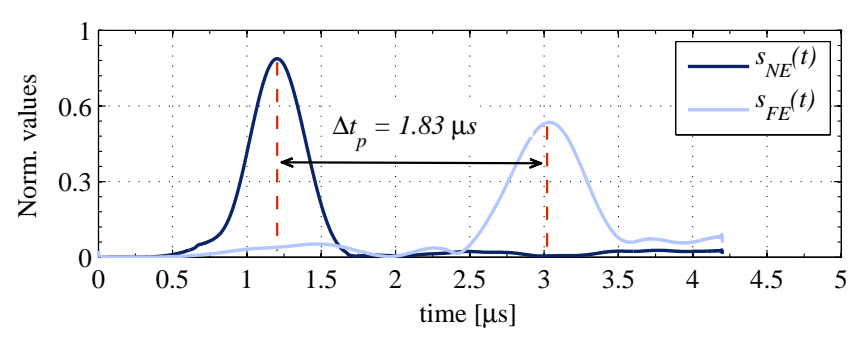

(b)

Figure 8: Example of measured PD signal: (a) near end signal, (b) baseband envelopes.

Figure 8(a) shows, as an example, the near end signal $x\left(t,-L_{1}\right)$, which exhibits a FWHM pulse width of $10.92 \mathrm{~ns}$ and a peak value of $200 \mathrm{mV}$. The resulting near and far end baseband signals are shown in Figure 8(b), where the estimated delay between them is also displayed. It is evident that $s_{F E}(t)$ is spread when compared to $s_{N E}(t)$.

The power of the baseband envelopes was estimated and, using (9), the attenuation constant was estimated to be $\alpha_{0}=$ 3.401e-3. The delay between these signals was also measured, and using $\left(\Delta t_{p}\right)$, the resulting delay constant was $\beta_{1}=$ $5.922 \mathrm{~ns} \mathrm{~m}^{-1}$. The resulting mean value of $\beta_{2}$ was $0.76 \mathrm{~ns}^{2} \mathrm{~m}^{-1}$, with a standard deviation of $0.19 \mathrm{~ns}^{2} \mathrm{~m}^{-1}$. Note that the proposed method is unable to estimate the sign of the dispersion constant, although it is easy to determine it.

Before the computation of $\beta_{2}, \sigma_{0}$ was estimated using (14), and the measured values of $\sigma_{N E}$ and $\sigma_{F E}$. The measured mean value of the initial deviation $\sigma_{0}$ was $1.79 \mathrm{~ns}$ with a standard deviation of $0.37 \mathrm{~ns}$ (see Figure 9(a)). In addition, the histograms of the measured deviation of the baseband signals shown in Figure 9(b) clearly exhibit the dispersion (or pulse spread) due to propagation. This histogram shows that the near end baseband signal is narrower (188 ns) than the far end one (229 ns).

\section{Discussion}

This section discusses some issues related to propagation that affect PD source location techniques accuracy in MV cables.

\subsection{Excessive attenuation at certain frequencies}

During the test campaign, some spectral components were observed to be highly attenuated at the far end. Additional factors, such as resonances or signal coupling, are responsible for some components to be extinguished. As a consequence, these components are not suitable to be used for PD source location. 


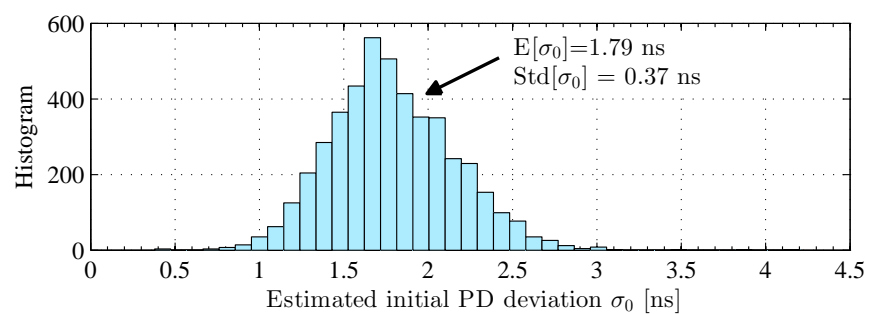

(a)

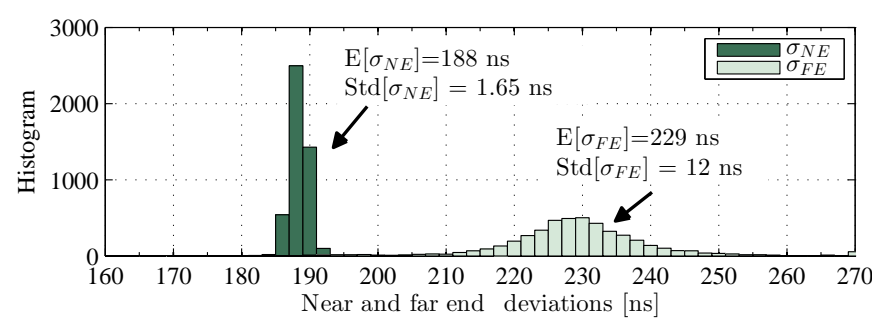

(b)

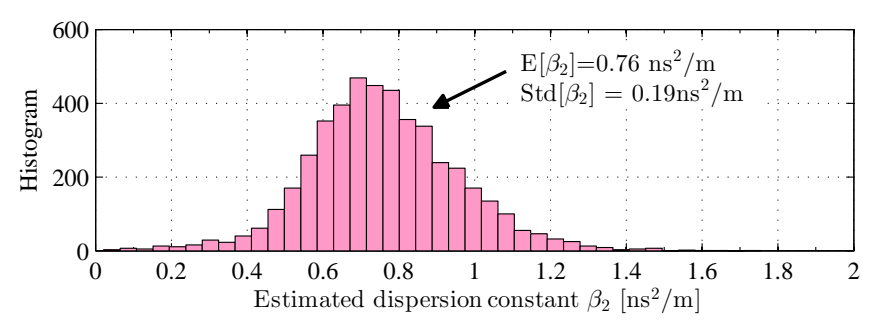

(c)

Figure 9: Experimental results: (a) histogram of the estimated PD initial deviation $\sigma_{0}$; (b) histograms of baseband signals envelope deviations $\sigma_{N E}$ and $\sigma_{F E}$; and (c) histogram of the measured dispersion constant $\beta_{2}$.

The method proposed in Figure 7 allows us to select the most suitable spectral components for source location.

\subsection{Attenuation and dispersion}

In [10] an expression for $A_{L}$ is proposed that takes into account the dissipative phenomenon that occurs in the cable. We propose an alternative expression for $A_{L}$ in (4), where we can distinguish two terms: a dissipative term, that depends on the attenuation constant $\alpha_{0}$, and a dispersive term, that depends on the dispersion constant $\beta_{2}$. Figure 10 depicts the evolution of both terms versus distance. We can observe that, when propagated distances are below $300 \mathrm{~m}$ (most of urban MV infrastructure), both terms are comparable. In those cases, the effect of dispersion in PD source location cannot be neglected.

\subsection{Attenuation dependence with frequency}

A PD is, in its early stage, a very fast phenomenon (it lasts for only a few nanoseconds, typically). Along the cable propagation, higher frequency components are attenuated due to conductor and dielectric losses, and particularly, due to the high frequency properties of the semiconducting layer. That is the reason why many papers in the literature model the real part of the propagation term as highly dependent on frequency (linearly in the case of [10]).

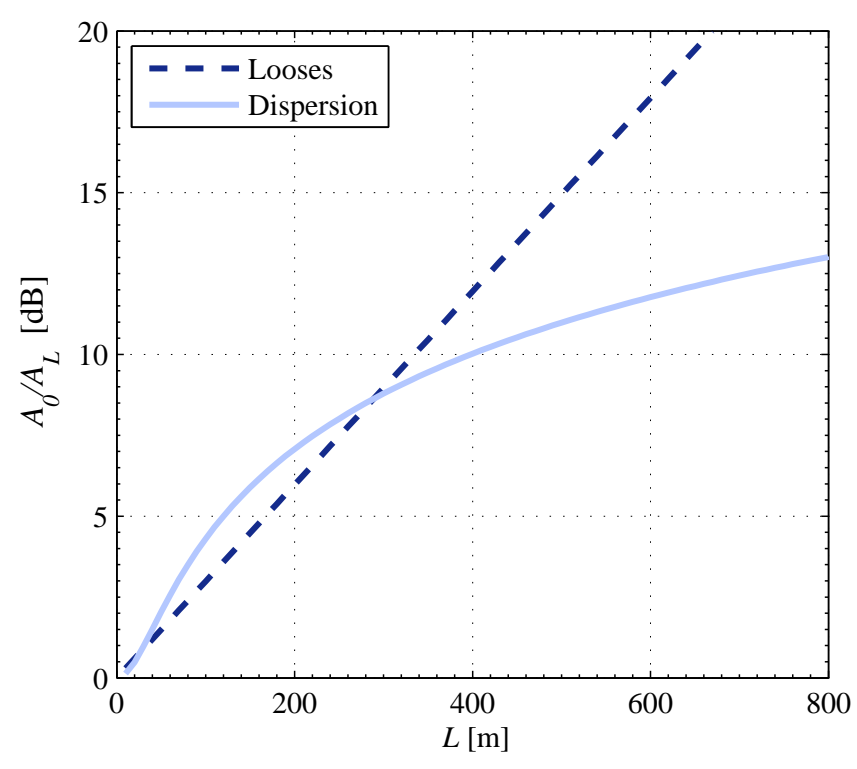

Figure 10: Peak value reduction due to attenuation and dispersion using a cable with $\alpha_{0}=3.44 \mathrm{e}-3 \mathrm{~m}^{-1}$ and $\beta_{2}=0.10 \mathrm{~ns}^{2} \mathrm{~m}^{-1}$.

The attenuation of higher frequency components due to propagation is responsible for the fact that only a small interval of the original PD spectrum reaches the cable ends where the PD sensors are installed. Although the attenuation constant is known to be dependent on frequency ([13]), an averaged value of the attenuation constant $\alpha_{0}$ can be computed in the bandwidth of interest. This averaging process should take into account the PD spectral components and the instrumentation, if they are known from actual measurements or can be estimated from previous measurements.

For example: in a XLPE cable 500 meters long with $\alpha_{1}=$ $4 \mathrm{e}-11 \mathrm{~s} / \mathrm{m}$, components located at $500 \mathrm{MHz}, 100 \mathrm{MHz}$ and 50 MHz would be attenuated $546 \mathrm{~dB}, 109 \mathrm{~dB}$ and $56 \mathrm{~dB}$, respectively. Then, the effective bandwidth of the captured PD signal useful for post-processing is limited to something less than 50 $\mathrm{MHz}$, although the bandwidth of the original signal is much greater in the origin. Even more, if the low pass behavior of the sensors is taken into account, the useful part of the spectrum will be further reduced. In this effective bandwidth we propose to simplify the attenuation to be frequency invariant.

\subsection{Delay and dispersion constants}

The VNA shows a negative dispersion constant in the measured spectral interval. Thus, the propagation velocity increases with frequency.

But the measured values for $\beta_{2}$ are so small that the expected variation in the propagation velocity is negligible from one spectral component to another (inside the captured bandwidth). In terms of propagation delay, two spectral components separated $\Delta f$ will be relatively delayed $\Delta t_{p}=2 \pi L \Delta f\left|\beta_{2}\right|$ where $L$ is the propagated distance. For example, using the measured $\beta_{2}$ in a $500 \mathrm{~m}$ long cable with $\Delta f=20 \mathrm{MHz}$, a $\Delta t_{p}$ of $6 \mathrm{~ns}$ is obtained. In terms of travelled distance, it is hardly one meter. This figure could make us to conclude that dispersion is 


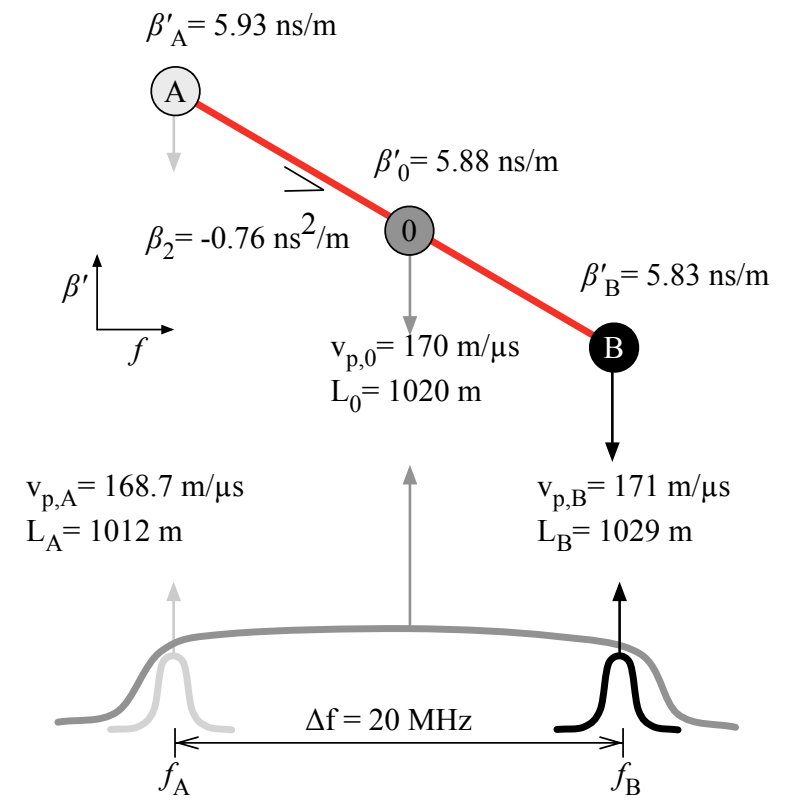

Figure 11: Example of propagation velocity variation due to non zero dispersion coefficient $\beta_{2}$.

irrelevant to PD source location.

We have observed that, in some frequency components, the phase term exhibits a significant non lineal behavior leading to a higher value of $\beta_{2}$. Then, the variation of the propagation velocity from one frequency component to another is not as small as it was in our experiment.

\subsection{Location errors due to propagation velocity variation}

From the point of view of location techniques (for example to assess the cable state in order to repair the degraded area), it is critical to know the precise value of the group delay $\beta_{1}$ for each frequency component used to estimate the overall delay, and thus, to compute the propagated distance.

The example shown in the diagram of Figure 11 depicts how varies $\beta^{\prime}$, the first derivative of the phase term $\beta$ versus frequency, in a spectral interval of $20 \mathrm{MHz}$. According to the proposed model, this variation is linear, and its slope is the dispersion constant $\left(\beta_{2}=-0.76 \mathrm{~ns}^{2} \mathrm{~m}^{-1}\right.$ in this example). Note that this diagram shows the group delay (inverse of the group velocity) for each frequency component.

In the frequency upper limit shown in Figure 11, labeled as $f_{B}, \beta_{B}^{\prime}$ is lower since the dispersion constant is negative, whereas in the lower boundary $\left(f_{A}\right)$ the group delay $\beta_{A}^{\prime}$ is greater. The equivalent propagation velocity for the three frequency components under study (labeled as A, B, and 0) are also shown in Figure 11. As a first approach, we can consider that the signals captured at the cable end (or cable ends) contain significant and similar spectral components in the whole frequency interval (this case is drawn in dark gray in Figure 11). Then, the propagation velocity will be $170 \mathrm{~m} / \mu \mathrm{s}\left(1 / \beta_{0}^{\prime}\right)$, that yields to an estimated distance $L_{0}=1020 \mathrm{~m}$ (for example for a given measured propagation delay of $6 \mathrm{~ns}$ ). Let us consider now that only a small part of the frequency components are available for location (in the extreme of the bandwidth, drawn in black and light gray in Figure 11). Such situation can arise either, because the rest of the components become extinct due to attenuation or destructive interference, or simply because an important amount of synchronous impulsive noise degrade these components. In these cases, the propagation velocity used to determine the estimated distance would be different $\left(v_{p, A}\right.$ or $\left.v_{p, B}\right)$, and the corresponding estimated distances $\left(L_{A}\right.$ and $\left.L_{B}\right)$ would be, for the same measured time delay, rather different to $L_{0}$.

The scheme proposed in Figure 7 allows us to select the most suitable frequency interval to be used for location purposes. And, for an accurate estimation of the fault location, the values of $\beta_{1}$ and $\beta_{2}$ provided by the model proposed in this paper are necessary.

\section{Conclusions}

This paper proposes a propagation model based on three parameters: one that models the signal attenuation, and two others that model its phase variation (the delay and the dispersion constants). When compared to other models, the proposed one exhibits similar peak and width values for both, originally symmetric and asymmetric pulses.

Two methods to estimate the model parameters are also proposed: starting from the classical frequency dependent attenuation constant described in the bibliography, or by using a vector network analyzer. Both methods provide similar results.

The main advantage of the proposed model is that the relationship between the propagation velocity and frequency, as well as the peak reduction and the PD spread due to dispersion, can be now quantified. We demonstrate that peak reduction due to dispersion cannot be underestimated, especially in urban distribution cables.

A method to estimate the dependence of the delay and dispersion constant with frequency is also proposed. In this method PD pulses are digitally processed in a narrow frequency interval. Using this technique we also obtained an estimation of the initial width of PD pulses in a XLPE cable.

The model and related techniques proposed in this paper give a more comprehensive view of PD propagation, contributing to a better understanding of this phenomenon and improving the accuracy of PD source location.

\section{References}

[1] R. Papazyan, R. Eriksson, Calibration for time domain propagation constant measurements on power cables, IEEE Trans. Instrum. Meas. 52 (2) (2003) 415-418

[2] P. Wagenaars, P. Wouters, P. Van Der Wielen, E. Steennis, Approximation of transmission line parameters of single-core and three-core xlpe cables, IEEE Transactions on Dielectrics and Electrical Insulation 17 (1) (2010) $106-115$.

[3] C. Herold, T. Leibfried, Advanced signal processing and modeling for partial discharge diagnosis on mixed power cable systems, IEEE Transactions on Dielectrics and Electrical Insulation 20 (3) (2013) 791-800.

[4] E. Ouatah, S. Megherfi, K. Haroun, Y. Zebboudj, Characteristics of partial discharge pulses propagation in shielded power cable, Electric Power Systems Research 99 (2013) 38-44. 
[5] J. Guo, S. Boggs, High frequency signal propagation in solid dielectric tape shielded power cables, IEEE Transactions on Power Delivery 26 (3) (2011) 1793-1802.

[6] A. Milioudis, D. Labridis, Modelling for on-line partial discharge monitoring on mv cables by using a modified universal line model, 2015.

[7] D. Clark, R. Mackinlay, R. Giussani, L. Renforth, R. Shuttleworth, Partial discharge pulse propagation, localisation and measurements in medium voltage power cables, 2013.

[8] E. Shu, S. Boggs, Dispersion and pd detection in shielded power cable, IEEE Electrical Insulation Magazine 24 (1) (2008) 25-29.

[9] J. Granado, C. Álvarez-Arroyo, A. Torralba, J. Rosendo-Macías, J. Chávez, M. Burgos-Payán, Time domain analysis of partial discharges envelope in medium voltage xlpe cables, Electric Power Systems Research 125 (2015) 220-227.

[10] N. Oussalah, Y. Zebboudj, S. c. Boggs, Partial discharge pulse propagation in shielded power cable and implications for detection sensitivity, IEEE Electrical Insulation Magazine 23 (6) (2007) 5-10.

[11] D. Marcuse, Pulse distortion in single-mode fibers, Appl. Opt. 19 (10) (1980) 1653-1660.

[12] High-voltage test techniques. partial discharge measurement, IEC 602702000.

[13] A. Morched, B. Gustavsen, M. Tartibi, A universal model for accurate calculation of electromagnetic transients on overhead lines and underground cables, IEEE Transactions on Power Delivery 14 (3) (1999) 1032-1037. 